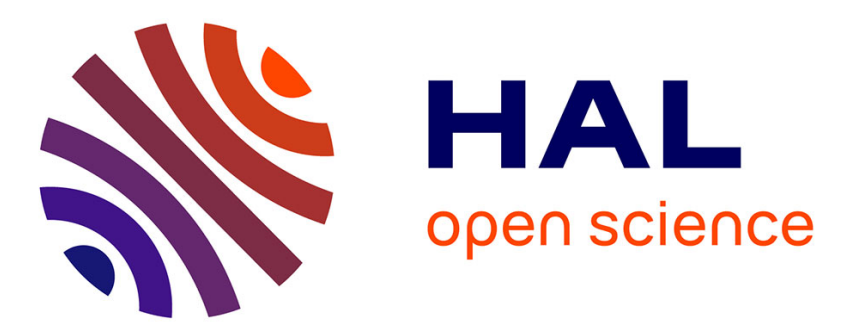

\title{
Diffusion-influenced deactivation of excited species in AOT microemulsions below, at, and above the percolation threshold
}

\author{
M. Almgren, R. Jóhannson
}

\section{- To cite this version:}

M. Almgren, R. Jóhannson. Diffusion-influenced deactivation of excited species in AOT microemulsions below, at, and above the percolation threshold. Journal de Physique IV Proceedings, 1993, 03 (C1), pp.C1-81-C1-90. 10.1051/jp4:1993108 . jpa-00251551

HAL Id: jpa-00251551

https://hal.science/jpa-00251551

Submitted on 1 Jan 1993

HAL is a multi-disciplinary open access archive for the deposit and dissemination of scientific research documents, whether they are published or not. The documents may come from teaching and research institutions in France or abroad, or from public or private research centers.
L'archive ouverte pluridisciplinaire HAL, est destinée au dépôt et à la diffusion de documents scientifiques de niveau recherche, publiés ou non, émanant des établissements d'enseignement et de recherche français ou étrangers, des laboratoires publics ou privés. 


\title{
Diffusion-influenced deactivation of excited species in AOT microemulsions below, at, and above the percolation threshold
}

\author{
M. ALMGREN and R. JÓHANNSSON \\ Department of Physical Chemistry, Uppsala University, P.O. Box 532, 75121 Uppsala, Sweden
}

\begin{abstract}
The deactivation of long-lived excited states by diffusive quenching has been examined for a microemulsion system, composed of small water droplets dispersed in an alkane solvent by means of the surfactant AOT. The reactants were confined to the water droplets, but with a possibility to exchange between the droplets - probably by the fusion and fission of individual droplets - giving rise to a slow deactivation process, following the initial fast deactivation from droplets which contained quenchers initially. From measurements using probes of different lifetime, and by varying the quencher concentration, it is possible to determine an average droplet size, to get some information about the polydispersity, to estimate the size and polydispersity of small clusters of micelles formed in dilute systems at low temperatures, and to determine the rate and character of the exchange processes between micelles in large and small clusters. In the interpretation of the decay data pertaining to the exchange between droplets in a cluster of droplets, the process was looked upon as a random walk over the cluster, leading to fractal type of decay for long walks over a loose cluster close to the percolation threshold, but to exponential decays both for the compact clusters at high water content, and for the short walks at high quencher concentration.
\end{abstract}


1. Introduction. - The phase behavior of ternary (quarternary) mixtures of ionic amphiphile - oil - water (+ inorganic electrolyte) has been discussed comprehensively and rationalized from the phase diagrams of the corresponding binary (or ternary) mixtures [1]. The most prominent feature in these phase diagrams - and the most well-studied one; for references to recent investigations see [2] - is a large microemulsion area, the $L_{2}$-phase, which extends from the oil corner to high contents of water and surfactant. With AOT as surfactant, where AOT stands for sodium-bis-(ethylhexyl)sulfosuccinate, the microemulsion consists of waterdroplets, solubilized in the oil by a layer of surfactant, which retain their integrity up to high water contents and temperatures (depending on the number of carbon atoms in the oil and the concentration of electrolyte). The size of the water pools has been determined, first by sedimentation ultracentrifugation $[3,4]$ and dynamic light scattering[49], later in numerous SANS studies[10-15], also by small angle X-ray scattering[16-18], and in fluorescence quenching experiments[19-23]. It is well established that the size depends little on temperature and solvent, and is determined mainly by the molar ratio of water to surfactant. The radius of the droplets is a linear function of this parameter, indicating that the aqueous core of the droplet is surrounded by a monolayer of surfactant molecules, each of which takes up a constant area at the interface. Some increase in size and polydispersity may occur when the temperature is raised, just before a critical demixing into two $\mathrm{L}_{2}$ phases takes place, but the effects observed in this region are probably mainly from critical fluctuations in droplet concentration and clustering of droplets, due to an increasingly attractive interaction between the droplets [24]. At high temperature and at a very high fraction of surfactant plus water, transition to a bicontinuous structure eventually takes place [2], but still at a volume fraction exceeding $60 \%$ for the dispersed phase the individual droplets have been found intact, but now with a well-defined nearest-neighbour ordering, and a liquid/glass-like transition [25-27] at a certain temperature.

Measurements of the electrical conductivity have revealed a percolative type of transition with increasing temperature [28-34], in regions where discrete droplets are known to exsist. Recently, Maitra et al.[35] showed that the water self-diffusion remained low far beyond the temperature or water volume fraction at which the conductivity went up. This supports the view of the conductance as mediated by the hopping of surfactant molecules from droplet to droplet when the droplets are linked together into long or fractal clusters due to the attractive interactions [31].

In fluorescence quenching, or more generally, in deactivation of excited species, a probe molecule is excited and dynamically deactivated by a second species, the quencher. Both are in this case hydrophilic species confined to the aqueous droplets. If the probe becomes excited in a droplet containing also quenchers, rapid deactivation will take place, and after a short period only probes which were excited in droplets without quenchers may remain excited. The fraction of the excited molecules which are characterized by this long lifetime, ideally equal to that of the probe in the absence of quenchers, can be determined, and assuming a random distribution (poissonian) of quenchers among the droplets, the number of droplets and hence their average size may be estimated. Variation of the estimated size with the quencher concentration gives a measure of the size polydispersity [36,37].

The more long-lived the probe, the more important will various exchange processes be for the slow decay following the initial intramicellar deactivation process. For AOT microemulsions it has been concluded that a dominant exchange mechanism is the fusionfission process, in which two droplets coalesce to form a large droplet, which then rapidly splits in two again $[22,23,38,39]$. In dilute microemulsions, this mechanism requires an encounter between two droplets, followed by the fusion. It has been found that in oils with low carbon number at room temperature, i.e. well away from the phase separation, the fission takes place in one of 100-1000 encounters. In systems approaching phase separation limit, however, the exchange processes become much faster and, when interpreted in the same way, indicate that fusion occurs on at least every encounter [22]. A better explanation is that under conditions when the attractive interdroplet interactions become important, the rapid exchange is from processes within preformed clusters of micelles [40]. Using a fairly long-lived triplet state for probe, it was shown clearly that in AOT - dodecane water, small clusters are present already in dilute microemulsions, and far below the 
clouding temperature. The characteristic decay obseved in these systems have three phases: (1) a rapid intramicellar deactivation, the rate of which depends on the size of the droplet; typically it is completed well within 100 nsec. (2) Deactivation of excited species formed in a droplet without quencher, but where the droplet is part of a cluster in which one or more quenchers are present. This process was observed to occur in the microsecond range. (3) Excited states produced in clusters without quenchers, show the natural decay, or are deactivated slowly through processes requiring exchange between clusters; these occur in the time range of $10-100 \mu \mathrm{s}$ [40].

The observed decay was modelled in correspondence to the Infelta - Tachiya model $[41,42]$ for fluorescence quenching in micellar solutions. The quenchers were assumed distributed according to a Poisson distribution among the clusters (an assumption which implies equal size of the clusters; in reality there is of course a broad distribution of cluster sizes, which makes the estimated apparent size strongly dependent on quencher concentration, as observed). The intermediate decay after the intramicellar process, due to quenching after exchange processes within the cluster, was assumed fully described by a first order quenching constant, $k_{c}$, which gives the quenching encounter frequency of a probe and a quencher in the same cluster, just as $\mathrm{k}_{\mathrm{q}}$ is used to describe the quenching in a micelle.

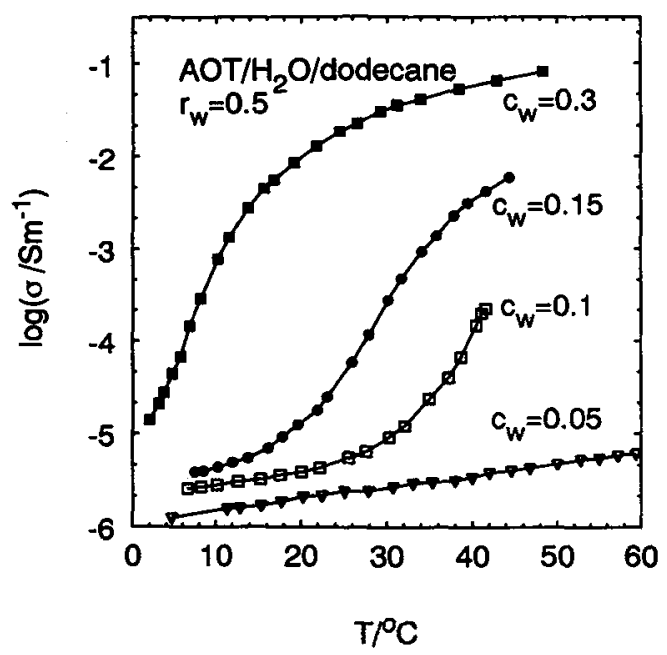

Fig. 1. - Conductivity versus temperature at various weight fractions of the dispersed phase in the system AOT-water-dodecane. Reproduced with permisssion from ref.[34]

The present contribution deals with clusters of droplets, and exchange processes in the clusters, for AOT - dodecane - water with increasing concentration of the dispersed phase. Sager et al.[34] have determined the conductivity at various temperatures for this system with results as reproduced in Fig. 1. The measurements refer to a weight ratio water to surfactant of 0.5 , corresponding to a molar ratio of 12.5. From the aggregation number of 160 , determined by the fluorescence quenching method, the aqueous droplet radius was calculated as $24 \AA$ in good agreement with earlier determinations. The conductivity in systems with a weight fraction above 0.1 of the dispersed phase increases strongly with temperature, but not as rapidly as in a typical percolation situation. We have chosen to study the deactivation of longlived excited states at a fixed temperature of $25^{\circ} \mathrm{C}$, at dispersed phase weight fractions, $c_{w}$, of $0.3,0.15$, and 0.075 , corresponding to points with extensive, probably rather compact clusters well above the percolation threshold, a fractal type of clusters close to the threshold, and only small clusters well below the threshold.

The previous model for deactivation in small clusters is not applicable at the percolation 
threshold or above. Even at very low quencher concentrations, the number of steps before quenching is limited, by practical reasons, to less than 30 . All clusters containing many more droplets than that will appear infinite, and an appropriate model is quenching by a random walk over an infinite cluster. Such a description will be discussed. For measurements at high quencher concentrations, or small clusters, different descriptions must be used. The relationship between the models and their applicability will be discussed. Experimental results for the different regimes will be presented and used to test the consistency of the description and to provide values for the parameters of the model, in particular the walk frequency, $\mathrm{k}_{\mathrm{w}}$.

2.1. Deactivation by random walk on a cluster. - Consider an excited probe $\mathrm{P}^{*}$ and quenchers $\mathbf{Q}$, present in the aqueous core of droplets gathered in a large cluster. The reactants may walk, by some mechanism, from droplet to droplet in the cluster; when they meet in the same droplet deactivation will occur. For simplicity the minority species, $\mathrm{P}^{*}$, is assumed stationary at the origin and the quenchers are assumed independent walkers with a walk frequency twice that of the real one. This is the target problem of Zumofen et al. [43], which is a good approximation to the situation when both reactants are free to walk. The quenchers are assumed distributed according to a Poisson distribution over the droplets.

The key quantity in this type of problems is $S_{n}$, the number of distinct sites visited in a walk of $n$ steps. Zumofen et al. [43] have shown that for euclidean lattices, and under conditions as given above, the survival probability of an excited probe, $\Phi_{n}$, is given exactly by

$$
\Phi_{\mathrm{n}}=\exp \left(-\mathrm{pS}_{\mathrm{n}}\right)
$$

where $p$ is the mean number of quenchers per droplet. $S_{n}$ has been calculated for many different types of lattices. For euclidean lattices the following results were quoted by Montroll and Weiss [44]:

$$
\begin{array}{ll}
\text { 1D: } & S_{n}=n^{1 / 2}(8 / \pi)^{1 / 2}+\ldots \\
\text { 2D: } & S_{n}=\pi n / 1 n n+\ldots \\
\text { 3D: } & S_{n}=n / P(0,1)+\ldots
\end{array}
$$

where $\mathrm{P}(0,1)$ is a constant depending on the latticec structure (1.5164...for a simple cubic, and $1.3445 \ldots$ for a body-centered cubic lattice. With an infinite number of neighbors to each site $P(0,1)$ should equal unity).

For irregular or fractal clusters the leading term of $S_{n}$ has been suggested to be given by $[45,46]$

$$
\begin{array}{ll}
S_{n}=a_{1} n^{d_{3}} & \text { for } d_{s}<2 \\
S_{n}=a_{2} n & \text { for } d_{s}>2
\end{array}
$$

where $d_{s}$ is the spectral or fracton dimension [47], and the prefactors $a_{1}$ and $a_{2}$ are unknown lattice-dependent constants, probably in the order of unity. For euclidean lattices $\mathrm{d}_{\mathrm{s}}=\mathrm{d}$, the euclidean dimension, and for a number of fractal lattices, including the percolating cluster at the pecolation threshold, $d_{s}$ has been found to be close to 4/3 [47]. Well above the percolation point, the clusters have a much more compact character (Swiss cheese, according to Stauffer [48]) and the spectral fractal dimension is expected to be above 2.

The leading term approximations for $S_{n}$ are only relevant for long walks - which means low quencher concentrations, $\mathrm{p}$ - and for those, time may be introduced as a continuous variable through

$$
\mathbf{n} \approx \mathbf{k}_{\mathbf{w}} \mathbf{t}
$$

where $\mathbf{k}_{\mathrm{w}}$ is the walk frequency. Noting that the observed signal is usually proportional to 
the concentration of excited species - or the survival probability - and that the excited state has a natural decay constant $k_{0}$, the final decay law is

with

$$
\mathrm{F}(\mathrm{t})=\mathrm{F}(0) \exp \left\{-\mathrm{pS}\left(\mathrm{k}_{\mathrm{w}} \mathrm{t}\right)\right\} \exp \left\{-\mathrm{k}_{0} \mathrm{t}\right\}
$$

$$
\begin{array}{ll}
S\left(k_{w} t\right)=a_{1}\left(k_{w} t\right)^{d_{s} / 2} & d_{s}<2 \\
S\left(k_{w} t\right)=a_{2}\left(k_{w} t\right) & d_{s}>2
\end{array}
$$

2.2. High quencher concentration $(p=1)$. - When the quencher concentration is high there is a high probability for quenching to occur in the next step. The decay expression presented above is no longer appropriate, for two reasons: (i) The expressions for $S_{n}$ only give the leading terms, valid for long walks. (ii) The time variable cannot be introduced with eq.(4); the time is not determined by the number of steps, but by the distribution of waiting times for the next step. In order to get a rate law a mean field argument can be used: the probability of quenching after the next step is proportional to the probability that at least one quencher, on average, will be present in the droplet after the next step, and little dependent of the history of the walk. We then have

$$
-d \Phi / d t=\Phi k_{w}\left(1-e^{-p}\right)
$$

which gives the following decay law

$$
F(t)=F(0) \exp \left\{-k_{w}\left(1-e^{-p}\right) t\right\} \exp \left\{-k_{0} t\right\}
$$

$\mathrm{F}(0)$ refers to the intensity after the initial intramicellar quenching event.

2.3. Small clusters. - Small clusters are fully explored after a certain time, i.e. after this time quenching has either occured, or will not occur at all. Probes excited in clusters without quenchers will give rise to an exponential final decay, ideally having the same decay constant as the unquenched probe. This situation was dealt with in a previous paper [40] as a generalization of the Infelta -Tachiya model for micellar quenching [41,42]. The decay law is

$$
F(t)=F(0) \exp \left\{-k_{0} t+p\left(e^{-k_{q} t}-1\right)+p(m-1)\left(e^{-k_{c} t}-1\right)\right\}
$$

where $m$ is the number of micelles per cluster (assumed constant). Since in reality the clusters probably display a broad distribution of sizes, the parameter values for $\mathbf{m}$ and $\mathbf{k}_{\mathrm{c}}$ obtained from fitting this equation to experimental data will depend on the quencher concentration.

The first-order cluster quenching constant $k_{c}$ refers to the encounter of a probe and a quencher in a cluster. If the quencher was not present in the droplet when the probe was excited, the probability that it will meet the probe after the first fusion event is given by $1 /(m-1)$. As a first crude approximation to $k_{c}$ we then have

$$
\mathbf{k}_{\mathrm{c}} \approx \mathbf{k}_{\mathrm{W}} /(\mathbf{m}-1)
$$

which would be useful when only very small clusters are present, besides free droplets. In this expression, $m$ must not be taken as an average over clusters and free droplets, but rather as an average over only real clusters. In practice, it may be used only to provide a lower limit to the walk frequency, $k_{w} \geq k_{c}$.

\section{Results and discussion.}

The experimental details of the investigation and a more comprehensive account of the 
results will be given elsewhere $[49,50]$.

3.1. Aggregation numbers and polydispersity of droplets. - To ensure that discrete droplets were present even in the most concentrated compositions, the aggregation numbers were determined with the dynamic fluorescence quenching method, using pyrenetetrasulfonate as probe and $\mathrm{I}^{-}$as quencher. In all cases the decays had welldevelopped exponential tails, with almost the same decay constant as the unquenched probe (with natural lifetime as short as $13.2 \mathrm{~ns}$ ), showing that closed droplets were present. The aggregation numbers were in good agreement with other determinations, corresponding to a water droplet radius of about $24 \AA$ at a water to surfactant mole ratio of 12.5 .

Since some discussion about the polydispersity has been on stage recently [51], the polydispersity was checked by measurements at several quencher concentrations $[36,37,50]$. It was found that $\sigma_{w} /\langle N\rangle_{w}$, the variance over the average of the weight distribution of the aggegation number, was about $40 \%$. The polydispersity is usually given with respect to the radius of the droplets. A closer inspection of the nature of the quenching average reveals that the value quoted corresponds to about $12 \%$ polydispersity of the radius [50], which result is much closer to that found by Ricka et al.[51] than the values given by several others, e.g.[52]. The low polydispersity also shows that there may hardly be a large population of merged droplets present, which implies that in the fusion-fission process the fusion is the rate-limiting step, followed after a short time by fission.

3.2. Deactivation dynamics in the concentrated system. - In the most concentrated system, $c_{w}=0.3$, two probe-quencher pairs were utilized, $\mathrm{Tb}(\mathrm{dpa})_{3}{ }^{3-}$ with ferroin as quencher, and $\mathrm{Cr}(\mathrm{bpy})_{3}{ }^{3+}$ with $\mathrm{NO}_{2}^{-}$as quencher. The former has a very long life-time, about $2 \mathrm{~ms}$, which makes measurements at very low quencher concentrations possible. The decays were always exponential in this regime, as expected from eqs. (5) and (7). The decay constants from the measurements are plotted in Figs. 2 and 3 vs. quencher concentration.

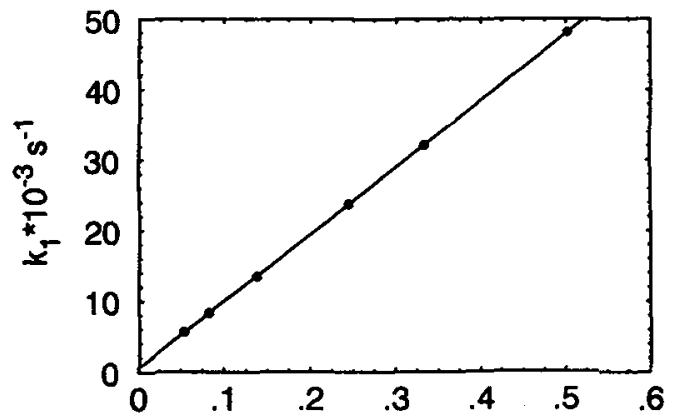

[Q] mmolal

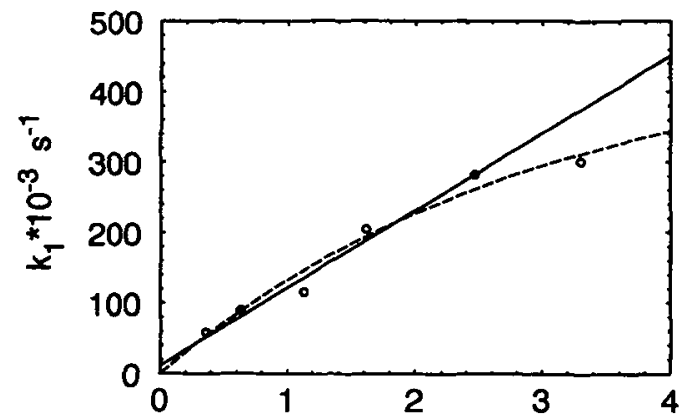

[Q] mmolal

Fig. 2. Decay constants of the exponential decays from quenching of $\mathrm{Tb}(\mathrm{dpa})_{3}{ }^{3-}$ by ferroin in microemulsions with $\mathrm{c}_{\mathrm{w}}=0.3$ at $25^{\circ} \mathrm{C}$

Fig. 3. Decay constants of the exponential decays from quenching of $\mathrm{Cr}(\mathrm{bpy})_{3}{ }^{3+}$ by $\mathrm{NO}_{3}{ }^{-}$in microemulsions with $c_{w}=0.3$ at $25^{\circ} \mathrm{C}$. The dashed curve represents $k_{w}\left(1-e^{-p}\right)$, where $p=$ [Q]/droplet], cf. eq.(9).

The results from measurements with $\mathrm{Tb}(\mathrm{dpa})_{3}{ }^{3-}$ produces a nice straight line, the slope of which provides a value for $a_{2} k_{w}=3.0 \times 10^{5} \mathrm{~s}^{-1}$, eqs. (5) and (7), which also fits reasonably well to the results at the lowest quencher concentrations with $\mathrm{Cr}(\mathrm{bpy})_{3}{ }^{3+}$. At higher quencher concentrations, however, the results from this probe deviates. The quencher concentrations are now in the regime where eq. (9) should apply, and as shown in Fig. 3 by 
the dashed curve, the expression $k_{w}\left(1-e^{-p}\right.$ ), with $k_{w}=4.7 \times 10^{5} \mathrm{~s}^{-1}$, reproduces the experimental decay constants reasonably well. Stretching the interpretation, these results give $\left(a_{2}\right)^{-1}=P(0,1) \approx 1.6$, a value somewhat larger than that for a simple cubic lattice, which is entirely reasonable.

3.3. Deactivation in the percolation threshold regime. - With $\mathrm{c}_{\mathrm{w}}=0.15$ at $25^{\circ} \mathrm{C}$ the conductivity, Fig. 1, is about halfway up (on the logarhitmic scale) to the high concentration plateau; open fractal clusters should still be predominant. Note that our measurements typically cover a time window of about 50 microseconds. With a step frequency of $3 \times 10^{5} \mathrm{~s}^{-1}$ the walks are not more than about 15 steps long. Under these conditions quite small clusters will appear infinite, and even if the correlation length of the infinite cluster has decreased considerably from the divergence at the percolation threshold, we have all reason to expect that it is long enough for $S_{n}$ to remain in the $d_{s} \approx 4 / 3$ regime.
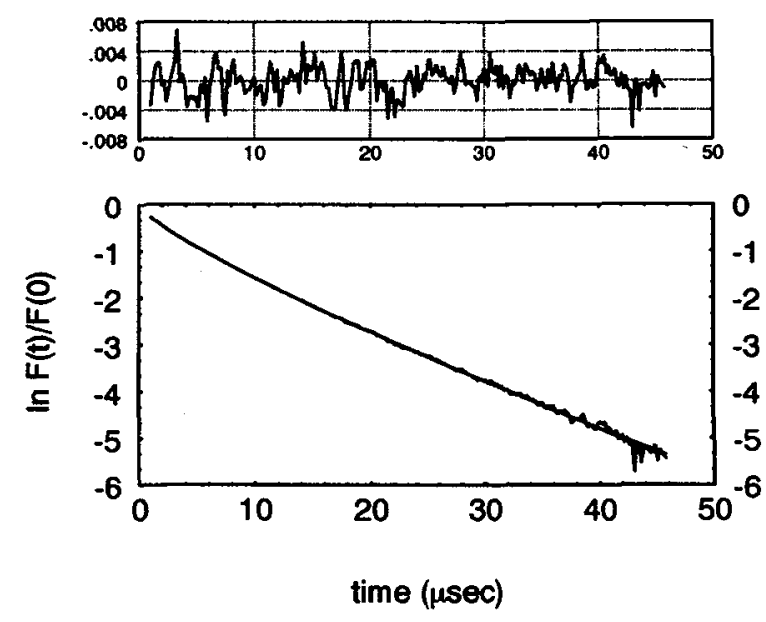

Fig. 4. The decay curve of $\mathrm{Cr}(\mathrm{bpy})_{3}{ }^{3+}$ quenched by $\mathrm{NO}_{3}{ }^{-}$in a microemulsion close to the percolation threshold, $c_{w}=0.15,25^{\circ} \mathrm{C}$. The upper curve shows the weighted residuals from a fit to the model of eqs. (5) and (6).

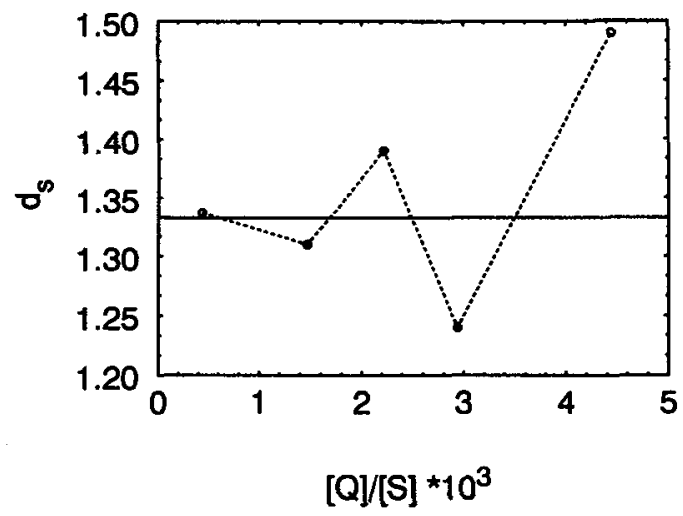

Fig. 5. The spectral dimension from fitting decay data of the type in Fig. 4 to eqs. (5) and (6). 
A typical decay curve for $\mathrm{Cr}(\mathrm{bpy})_{3}{ }^{3+}$, quenched by $\mathrm{NO}_{3}{ }^{-}$, is shown in Fig. 4. The decay is non-exponential, and fits well to the model defined by eqs.(5) and (6). The values obtained for the spectral dimension from measurements at several quencher concentrations are shown in Fig. 5 to be distributed around the value of 4/3. By constraining $d_{s}$ to its mean value of 1.36 , the value of $\mathrm{a}_{1}{ }^{2 / \mathrm{d}_{\mathrm{s}}} \mathrm{k}_{\mathrm{w}}$ was obtained as $4.0 \times 10^{5} \mathrm{~s}^{-1}$, in the same range as the estimate of $\mathrm{k}_{\mathrm{w}}$ from the high concentration regime.

The results from the measurements at high and medium droplet concentrations are thus internally consistent.

3.4. Small clusters at low droplet concentration. - Measurements were performed with $c_{w}=0.15$ at $15^{\circ} \mathrm{C}$, and with $c_{w}=0.075$ at 25 and $12{ }^{\circ} \mathrm{C}$ giving decay curves as the one shown in Figure 6, which fitted well to eq. (10). The results indicate that small clusters are present, with $m \approx 2$. The value for the first-order deactivation constant $k_{c}$, which should be equal to or smaller than $\mathrm{k}_{\mathrm{w}}$, was obtained as $1.7 \times 10^{5} \mathrm{~s}^{-1}$ at $\mathrm{c}_{\mathrm{w}}=0.15,15^{\circ} \mathrm{C}$, and $2.1 \times 10^{5} \mathrm{~s}^{-1}$ at $c_{w}=0.075,25^{\circ} \mathrm{C}$. These results suggest a value of $k_{w}$ somewhat lower than at the highest concentration of droplets, which well may be a real effect; in the compact clusters each micelle have many neighbours and would be expected to undergo fusion processes with higher frequency than in a small cluster with only one or two neighbours.
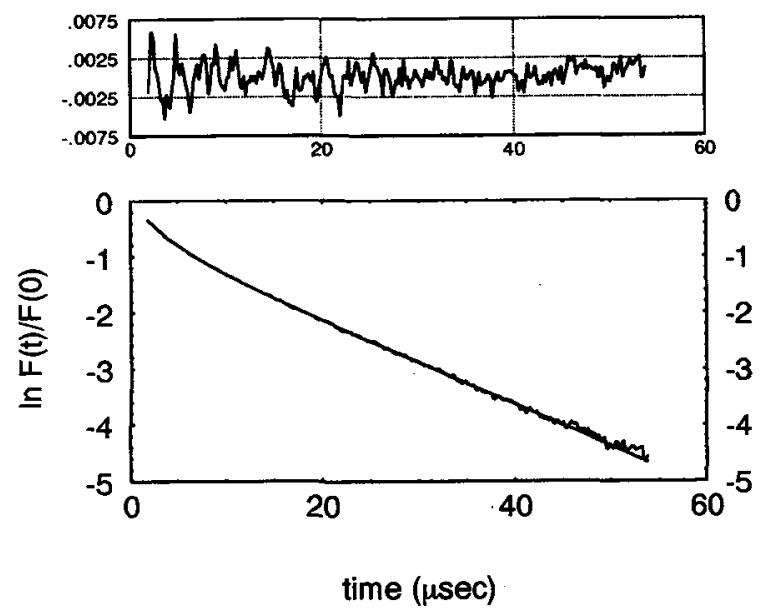

Fig. 6. Decay curve from the dilute regime, $c_{\mathrm{w}}=0.075,25^{\circ} \mathrm{C}$, with residuals from a fit to eq.(10)

4. Conclusions. - The experimental decay curves are in qualitative accord with the expected behaviour in the various regimes, and produce consistent values of the pertinent rate parameter, $\mathrm{k}_{\mathrm{w}}$, and close to the percolation threshold, a value of the spectral dimension in the expected range. The fact that the deactivation proceeds at the same rate for a number of different probe - quencher pairs supports the notion of a mechanism for exchange which is independent of the nature of the reactants, a likely candidate being the fusionfission mechanism. ( $\mathrm{I}^{-}$as quencher is an exception, giving a faster deactivation by almost an order of magnitude than the other quenchers [40]). - A number of questions present themselves, however, on more detailed considerations: (i). The walks considered are mainly quite short, in the order of 10-20 steps - is this enough for the use of only the leading terms in the expressions for $S_{n}$ ? (ii). With fusion-fission as the transport mechanism the cluster itself is changing during the walk - what is the effect on the spectral dimension?

Acknowledgement. - This work was supported by the Swedish Natural Science Research Council. 


\section{References}

[1] Kahlweit, M, Strey, R, Schomacker, R, Haase, D, Langmuir 5 (1989) 305

[2] Chen, S-H, Chang, S-L, Strey, R, J. Chem. Phys. 93 (1990) 1907

[3] Eicke, H F, Rehak, J, Helv. Chim. Acta 59 (1976) 2883

[4] Robinson, B H, Steyler, D C, Tack, R D, J. Chem. Soc., Faraday Trans. I 75 (1979) 481

[5] Zulauf, M, Eicke, H F, J. Phys. Chem. 83 (1979) 480

[6] Sein, E, Lalanne, J R, Buchert, J, Kielich, S, J. Colloid Interface Sci. 72 (1979) 363

[7] Day, R A, Robinson B H, Clarke, J H R, Doherty, J V, J. Chem. Soc., Faraday Trans. I 75 (1979) 132

[8] Gulari, E, Bedwell, B, Alkhafaji, S, J. Colloid Interface Sci. 77 (1980) 202

[9] Clarke, J H R, Nicholson, J D, Regan, K N, J. Chem. Soc., Faraday Trans. I 81 (1985) 1173

[10] Cabos, C, Delord, P, J. Appl. Crystallogr. 12 (1979) 502

[11] Robinson, B H, Toprakcioglu, C, Dore, J C, Chieux, P, J. Chem. Soc., Faraday Trans. I 80 (1984) 13

[12] Toprakcioglu, C, Dore, J C, Robinson, B H, Howe, A, J. Chem. Soc., Faraday Trans. I $80(1984) 413$

[13] Kotlarchyk, M, Chen, S H, Huang, J S, J. Phys. Chem. 86 (9182) 3273

[14] Kotlarchyk, M, Chen, S H, Huang J S, Kim, M W, Phys. Rev. A 29 (1984) 2054

[15] Kotlarchyk, M, Huang, J S, Chen, S H, J. Phys. Chem. 89 (1985) 4382

[16] Assih, T, Larché, Deloed, P, J. Colloid Interface Sci. 89(1982) 35

[17] Cabos, C, Marignan, J, J. Phys. Lett. 46 (1985) L-267

[18] Pileni, M P; Zemb, T, Petit, C, Chem. Phys. Lett. 118 (1985) 414

[19] Atik, S S,. Thomas, J K, J. Am. Chem. Soc. 103 (1981) 3543

[20] Bridge, N J, Fletcher, P D I, J. Chem. Soc., Faraday Trans. I 79 (1983) 2161

[21] Ganz AM, Boeger, B E, J. Colloid Interface Sci. 109 (1986) 504

[22] Lang J, Jada, A, Malliaris, A, J. Phys. Chem. 92 (1988) 1946

[23] Howe, A H, McDonald, J A, Robinson, B H, J. Chem. Soc., Faraday Trans. I 83 (1987) 1007

[24] Chen, S H; Lin, T L, Kotlarchyk, M, in"Surfactants in Solution", Mittal, K L, Bothorel, P, eds., Plenum Press, New York Vol.6 (1986) 1315

[25] Kotlarchyk, M, Chen, S H, Huang J S, Kim, M W, Phys. Rev. Lett. 53 (1984) 941

[26] Kotlarchyk, M, Huang J S, Kim, M W, Chen, S H, in"Surfactants in Solution", Mittal, K L, Bothorel, P, eds., Plenum Press, New York Vol.6 (1986) 1303

[27] Huang, J S, in "Surfactants in Solution", Mittal, K L, ed., Plenum Press, New York, Vol. 10 (1989) 45

[28] Lagues, M, Ober, R, Taupin, C, J. Phys. Lett. 39 (1978) 487

[29] Cazabat, A M, Chatenay, D, Langevin, D, Meunier, J, Faraday Discuss. Chem. Soc. 76 (1982) 291

[30] Battacharya, S, Stokes, J, Kim, M W, Huang, J S, Phys. Rev. Lett. 55 (1985) 1884

[31] Kim, M W, Huang, J S, Phys. Rev. A34 (1986) 714

[32] Peyrelasse, J, Boned, C, Marin, G, Colloid Polymer Sci. 264 (1986) 143

[33] Borkovec, M, Eicke, H-F, Hammerich, H, Das Gupta, B, J. Phys. Chem. 92 (1988) 206

[34] Sager, W, Sun, W, Eicke, H-F, Progr. Colloid Polymer Sci in press 1992

[35] Maitra A, Mathew, C, Varshney, M, J. Phys. Chem. 94 (1990) 5290

[36] Almgren, M, Löfroth, J-E, J. Chem. Phys. 76 (1982) 2734

[37] Warr, G G, Grieser, F G, J. Chem. Soc., Faraday Trans. I 82 (1986) 1825

[38] Fletcher, P D I, Howe A M, Robinson, B H, J. Chem. Soc., Faraday Trans. I 83 (1987) 185

[39] Fletcher, P D I, Robinson, B H, Ber. Bunsenges. Phys. Chem. 85 (1981) 857

[40] Jóhannsson, R, Almgren, M, Alsins, J, J. Phys. Chem. 95 (1991) 3819

[41] Infelta, P P, Grätzel, M, Thomas, J K, J. Phys. Chem. 78 (1974) 190

[42] Tachiya M, Chem. Phys. Lett. 33 (1975) 289

[43] Zumofen, G, Blumen A, Klafter, J, J. Chem. Phys. 82 (1985) 3198

[44] Montroll, E W, Weiss, G H, J. Math. Phys.6 (1965) 167

[45] Rammal, R, Toulouse, G, J. Physique Lett. 44 (1983) L-13 
[46] Blumen, A, Klafter, J, Zumofen, G, Phys. Rev. B 28 (1983) 6112

[47] Alexander, S, Orbach, R, J. Physique Lett, 43 (1982) L-625

[48] Stauffer, D, Phys. Reports, 54 (1979) 1

[49] Almgren, M, Johannsson, R, J. Phys. Chem. (submitted)

[50] Johannsson, $R$, Almgren, $M$, to be published

[51] Ricka, J, Borkovec, M, Hofmeier, U, J. Chem. Phys. 94 (1991) 8503

[52] Kotlarchyk, M, Stephen, R R, Huang, J S, J. Phys. Chem. 92 (1988) 1533 